\title{
Manipulation of the magnetron orbit of a positron cloud in a Penning trap
}

\author{
T. Mortensen, ${ }^{1, a)}$ A. Deller, ${ }^{1}$ C. A. Isaac, ${ }^{1}$ D. P. van der Werf, ${ }^{1}$ M. Charlton, ${ }^{1}$ \\ and J. R. Machacek ${ }^{2}$ \\ ${ }^{1}$ Department of Physics, College of Science, Swansea University, Singleton Park, Swansea SA2 8PP, \\ United Kingdom \\ ${ }^{2}$ Centre for Matter-Antimatter Studies, Physics Laboratories, Research School of Physics and Engineering, \\ Australian National University, Canberra ACT 0200, Australia
}

(Received 20 November 2012; accepted 15 January 2013; published online 31 January 2013)

\begin{abstract}
We describe a simple and versatile method to manipulate the amplitude of the magnetron orbit of ions stored in a Penning trap, applied here to a cloud of low energy positrons. By applying a pulsed voltage to a split electrode in the trap, which is normally used for rotating wall compression of the particles, the size of the magnetron orbit can be changed at will. The modified orbit has been shown to be stable for many magnetron periods. The technique could find use in applications which require off-axis ejection of particles, for instance in the filling of arrays of traps for multicell positron storage. (C) 2013 American Institute of Physics. [http://dx.doi.org/10.1063/1.4789880]
\end{abstract}

\section{INTRODUCTION}

Penning type traps have found numerous applications in both physics and chemistry ranging from the formation of antihydrogen $^{1-3}$ to highly sensitive mass spectrometry. ${ }^{4}$ These traps are capable of operation with a wide range of charged particle numbers, from clouds consisting of just a few particles, ${ }^{5}$ the so-called single particle regime, to plasmas where the density is sufficiently high that their mutual interactions cannot be neglected and collective effects emerge. ${ }^{6}$

In recent years, it has become feasible to manipulate the radial extent of both clouds and plasmas through the application of the so-called rotating wall (RW) technique, ${ }^{7-14}$ though the mechanism responsible for the effect varies between the two regimes. Here, one of the confining electrodes (see Sec. II) is azimuthally split, typically into four or eight segments, and appropriate phase shifted time-varying voltages are applied to each part. The resulting dipolar or quadrupolar electric field is arranged to rotate in the same sense as the natural rotation of the plasma or the magnetron motion of the particles in the cloud, and the torque thus imparted causes the plasma or cloud to compress. It is usually necessary to counteract the heating caused by the field, and this is commonly achieved by the introduction of a cooling gas into the system.

RW compression reduces particle loss via diffusion to the electrode walls of the $\operatorname{trap}^{8}$ and has found widespread use, for instance in tailoring antiparticle plasmas and clouds for antihydrogen $^{15-21}$ and antiproton ${ }^{22,23}$ physics, and in manypositron experimentation. ${ }^{24,25} \mathrm{~A}$ possible application is in the development of multicell traps for the storage of large numbers of positrons, ${ }^{26,27}$ where it is envisaged that an array of microtraps will be filled from a positron accumulator (see e.g., Refs. 28 and 29) for long term storage and later use. Such devices require the traps in the array to be independently addressable. Since the RW technique always compresses the plasma or cloud to the centre of the trap, methodology is

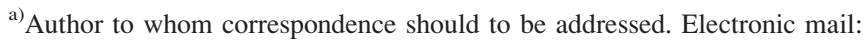
T.Mortensen.369921@Swansea.ac.uk.
}

required to move the ensemble radially in a controlled and reproducible manner.

In the plasma regime, excitation of the diocotron mode, ${ }^{30,31}$ coupled with frequency phase locking via autoresonant excitation of the mode, ${ }^{32}$ can be used to achieve radial displacement, as has been demonstrated in the validation of the multicell concept by Surko and co-workers. ${ }^{26}$ However, in the single particle (non-interacting) regime, the diocotron mode does not exist, and a different approach must be sought.

We have found that applying an electric field radially across a compressed cloud of positrons for a short time can initiate an orbit which may be described using single-particle dynamics and the radius of which can be controlled with precision. The technique has similarities to, though is distinct from, the so-called sidekick method used in ion cyclotron resonance mass spectrometry ${ }^{33,34}$ to enhance the capture efficiency and increase resolving power. The remainder of the article is organised as follows: Sec. II gives a summary of pertinent theoretical and experimental details, Sec. III contains our results and discussion, with Sec. IV presenting concluding remarks.

\section{THEORETICAL AND EXPERIMENTAL DETAILS}

It is worth recalling some of the basic physics of the Penning trap. ${ }^{5}$ Cylindrically symmetric electrodes are used to produce a potential extremum in the centre of the trap which, in conjunction with an axial applied magnetic field, $\mathbf{B}=B \hat{\mathbf{z}}$, results in stable confinement of charged particles The electric potential experienced by a charged particle in an ideal trap is given by the first term of the following equation:

$$
\phi=\frac{m}{q} \frac{\omega_{z}^{2}}{2}\left(z^{2}-\frac{r^{2}}{2}\right)+\frac{m}{q} a x,
$$

where $q$ and $m$ are the charge and the mass of the particle, respectively, $r$ is the radial distance from the trap centre, and $z$ is the axial co-ordinate. The value of the so-called axial bounce frequency, $\omega_{z}$, depends on the electrode geometry and the applied voltages. More pertinent here are the radial 
motions. In a constant magnetic field and with zero perpendicular electric field, charged particles orbit the field axis at the cyclotron frequency, $\Omega_{c}=q B / m$. The application of a Penning trap potential (i.e., the first term in Eq. (1)) gives rise to $\mathbf{E} \times \mathbf{B}$ fields which modify $\Omega_{c}$ into $\omega_{+}$and cause the particle to exhibit magnetron motion with a frequency $\omega_{-}$. These frequencies are given by $\omega_{ \pm}=\frac{1}{2}\left(\Omega_{c} \pm \sqrt{\Omega_{c}^{2}-2 \omega_{z}^{2}}\right)$. For the experiment described herein, the magnetic field inside the trap is $38 \mathrm{mT}$, and the motional frequencies are estimated to be $\omega_{+} \approx 6.6 \mathrm{Grad} \mathrm{s}^{-1}, \omega_{z} \approx 60 \mathrm{Mrad} \mathrm{s}^{-1}$, and $\omega_{-} \approx 270 \mathrm{krad} \mathrm{s}^{-1}$.

For a single particle located on-axis, application of a dipole field in the radial plane of the trap will induce decentred orbital motion which persists for the duration of the applied pulse. Removal of the field at time $t_{1}$ results in an orbit about the trap centre with a radius of $d_{M}\left(t_{1}\right)$ derived below.

The additional field applied in the $x$-direction gives the second term in Eq. (1), where $a$ is a trap-dependent parameter (see below). This term modifies the classic Penning trap equations of motion to become

$$
\ddot{z}=-\omega_{z}^{2} z, \quad \ddot{x}=\frac{\omega_{z}^{2}}{2} x+\Omega \dot{y}-a \quad \text { and } \quad \ddot{y}=\frac{\omega_{z}^{2}}{2} y-\Omega \dot{x} .
$$

By defining $u=x+i y$, the last two equations in Eq. (2) can be combined to give

$$
\ddot{u}+i \Omega \dot{u}-\frac{\omega_{z}^{2}}{2} u=-a,
$$

which has the general solution

$$
u(t)=c_{+} e^{-i \omega_{+} t}+c_{-} e^{-i \omega_{-} t}+k,
$$

with $k=2 a / \omega_{z}^{2}$. Applying the initial conditions $u(0)=\dot{u}(0)$ $=0$ fixes $c_{+}$and $c_{-}$as

$$
c_{ \pm}= \pm k \frac{\omega_{\overline{+}}}{\omega_{+}-\omega_{-}} .
$$

The total radial displacement, which assuming $c_{+}$and $c_{-}$are real, is given by

$$
\begin{aligned}
|u|^{2}= & c_{+}^{2}+c_{-}^{2}+k^{2}+2 c_{+} k \cos \left(\omega_{+} t\right)+2 c_{-} k \cos \left(\omega_{-} t\right) \\
& +2 c_{+} c_{-} \cos \left(\left[\omega_{+}-\omega_{-}\right] t\right) .
\end{aligned}
$$

Applying the approximations $c_{-} \approx-k$ and that $c_{-}, k \gg c_{+}$, which are justified from the expressions given above since $\omega_{+} \gg \omega_{-}$, simplifies Eq. (6) to give an approximate expression for the displacement, $d_{M}(t)$, as

$$
d_{M}(t) \approx \frac{2 \sqrt{2} a}{\omega_{z}^{2}} \sqrt{1-\cos \omega_{-} t}=2 k\left|\sin \left(\omega_{-} t / 2\right)\right| .
$$

Thus, we find that the displacement is governed by the magnitude and direction of the applied dipole and undergoes oscillatory behaviour at an angular frequency of $\omega_{-}$.

Applying this field can also be interpreted as moving the radial centre of the trap. This can easily be demonstrated by rewriting Eq. (1) as

$$
\begin{aligned}
\phi & =-\frac{m \omega_{z}^{2}}{4 q}\left(-2 z^{2}+x^{2}+y^{2}\right)+\frac{m}{q} a x \\
& =-\frac{m \omega_{z}^{2}}{4 q}\left(-2 z^{2}+\left(x-\frac{2 a}{\omega_{z}^{2}}\right)^{2}+y^{2}\right)+\frac{a^{2} m}{q \omega_{z}^{2}}
\end{aligned}
$$

Thus, a transform of the form $x \rightarrow(x-k)$ will return the standard Penning potential with an added constant.

The above discussion assumes instantaneous application (and later removal) of a pulsed field; indeed, in practice, it is necessary that the dipole be switched on in a time much shorter than the magnetron period, breaking an adiabatic invariant $^{35}$ and instigating the preliminary orbital motion (green broken line, Figure 1(a)). Equivalently, in order that the cloud will follow the ultimate orbital path (red dotted line, Figure 1(a)), the additional field must be removed just as rapidly.

The experiment was conducted in a two-stage positron accumulator which has been described in detail elsewhere. ${ }^{29}$ The second stage of this instrument is comprised of five electrodes, each $49 \mathrm{~mm}$ long with an internal diameter of $41 \mathrm{~mm}$. One of these electrodes is divided into two, with one of the halves cut azimuthally into four segments. This electrode is used to RW compress the positron cloud, as described by Isaac and co-workers. ${ }^{13,14} \mathrm{~A}$ schematic of the second stage of the trap is given in Figure 2.

Compressed clouds of $\sim 10^{5}$ positrons were produced in a two-step procedure: (i) the voltage applied to the entrance electrode of the first stage of the trap was lowered to allow positrons to enter. Collisions with nitrogen gas in the first and second stages resulted in the positrons residing in the latter after a few $\mu$ s or so. ${ }^{28}$ During accumulation, the rotating wall was switched on, thus compressing the cloud in the presence of the $\mathrm{CO}_{2}$ cooling gas; (ii) after a predetermined accumulation period, the entrance electrode voltage was raised to prevent further particles from entering, and the rotating wall was switched off. The positron clouds have a radius of approximately $1 \mathrm{~mm}$, with a temperature estimated to be $900 \mathrm{~K}$, as measured by use of the method developed by Eggleston et $a l .{ }^{36}$ A self-consistent potential solver was used to determine the cloud length and density as $6 \mathrm{~mm}$ and $5 \times 10^{12} \mathrm{~m}^{-3}$, respectively. The Debye length $\left(\lambda_{d}\right)$ of such a cloud is $\sim 1 \mathrm{~mm}$, which does not satisfy the plasma regime inequality, $\lambda_{d} \ll r_{\text {plasma }}$, though as such may be considered a rarified plasma ${ }^{37}$ and some collective phenomena (shielding, waves, and global thermal equilibria) accordingly presumed. Moreover, although we may explain the following observations from an ideal, single-particle perspective, the collective effect of self-rotation likely inhibits shearing of the cloudsotherwise expected in less dense collections, as a consequence of the non-ideal trapping potential which results in small differences in the magnetron frequency across the cloud diameter.

Following these procedures, at time $t=0$ (see Figure 1(a)), additional bias voltages of $\pm V_{b}$ were applied to opposing segments of the four-way split electrode $\left(\left|V_{b}\right|<1 \mathrm{~V}\right)$, creating an approximate dipole field across the cloud and projected near uniformly along its length, distorting the 
a)

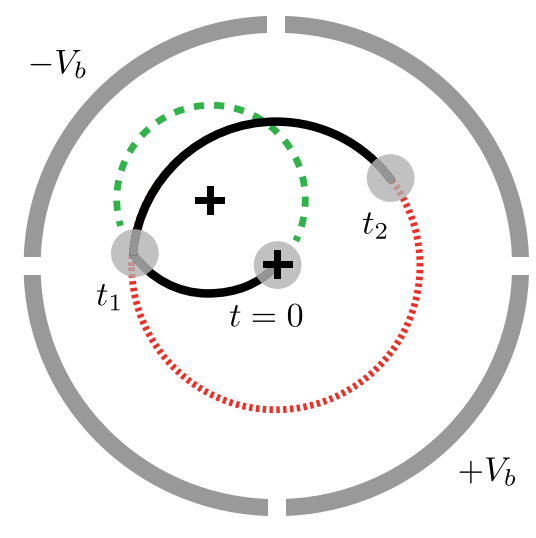

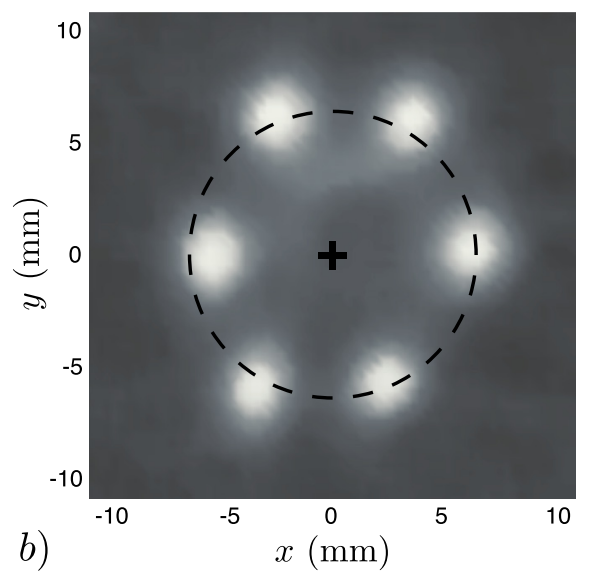

FIG. 1. (a) Schematic radial cross section of the RW electrode showing the bias voltages applied to opposing segments. The black solid line is the path of the positron cloud (grey disk) during the experiment (see text). The green broken line represents the cloud orbit during application of the bias voltages. The red dotted line follows the orbit after the bias voltages are removed. (b) Combined picture of positron clouds for a number of different positions within one magnetron orbit after a bias voltage of $0.36 \mathrm{~V}$ was switched on for $7 \mu$ s. The dashed line is a fit of a circle to the magnetron orbit with the cross denoting the centre of the trap. Asymmetries in the transport fields are the likely cause of the slight offsets of the clouds from the fitted orbit.

trapping potential and shifting the extremum, as described above (illustrated by the crosses in the Figure 1(a)). This results in the cloud following the orbit depicted by the green broken line. When at $t_{1}$ the bias is switched off $\left(3 \mu \mathrm{s} \leq t_{1}\right.$ $\leq 45 \mu \mathrm{s}$ ), the trapping potential reverts to the original, leaving the cloud circling about the centre of the trap (red dotted line). At time $t_{2}$, the cloud is released, by rapidly (within a time of $\sim 20 \mathrm{~ns}$ ) lowering the voltage applied to the end electrode of the trap, and then dumped onto the microchannel plate (MCP)-phosphor screen assembly (see Figure 2). The resulting phosphorescence was recorded with a CCD camera and the image fitted with a 2D-Gaussian to determine the location of the cloud centre and its width. It should be noted that the magnetic field at the MCP-phosphor screen position is lower by a factor of 6.6 with respect to the trapping field, such that the radii of the clouds inside the trap are smaller by a factor of 2.6 compared to that measured on the screen. The fitted coordinates and widths of the clouds produced by this method were found to be reproducible with a standard deviation of about $0.15 \mathrm{~mm}$ and $0.03 \mathrm{~mm}$, respectively. As an example, Figure 1(b) shows a compendium of cloud images for a number of different positions (i.e., a number of different values for $t_{2}$ ) within one magnetron orbit.

The cloud widths slowly expand with time due to collisions with the trapping and cooling gases in the second

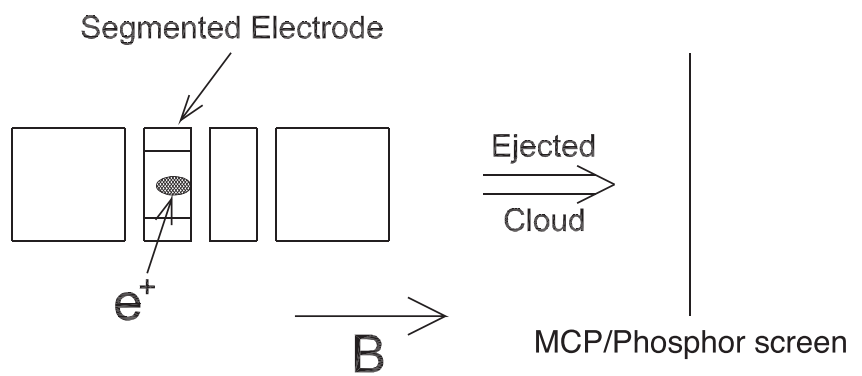

FIG. 2. Schematic of part of the accumulator second stage with a segmented electrode and the MCP-phosphor screen system used to measure the radial position of the ejected positron cloud. The axial magnetic field, B, is provided by an external solenoid. Phosphor screen images were recorded by an external CCD camera. stage. ${ }^{14}$ The gas pressures were selected to provide optimum compression and lifetime as opposed to reducing expansion. However, the manipulation of the magnetron orbits reported here occurs on a much shorter timescale than that of expansion, and as such many orbits can be observed before the cloud is no longer clearly distinguishable. (The cloud was found to expand at rate of around $32 \mathrm{~mm} \mathrm{~s}^{-1}$, equivalent to an increase in width of about $0.68 \mu \mathrm{m}$ during one magnetron orbit.)

\section{RESULTS AND DISCUSSION}

Magnetron radii and frequencies were obtained by fitting the $x$ and $y$ coordinates of the imaged cloud for varying particle hold times $\left(t_{2}-t_{1}\right)$, and an example is shown in Figure 3 . The fitted frequency $(47.3 \pm 0.1 \mathrm{kHz})$ for the data in Figure 3 is roughly in accord with the expected ideal trap magnetron orbital frequency $\left(\omega_{-} / 2 \pi\right)$ of $43 \mathrm{kHz}$, as calculated from the magnetic field and trapping potential. This is also consistent with resonant particle ejection at $\approx 46 \mathrm{kHz}$, as

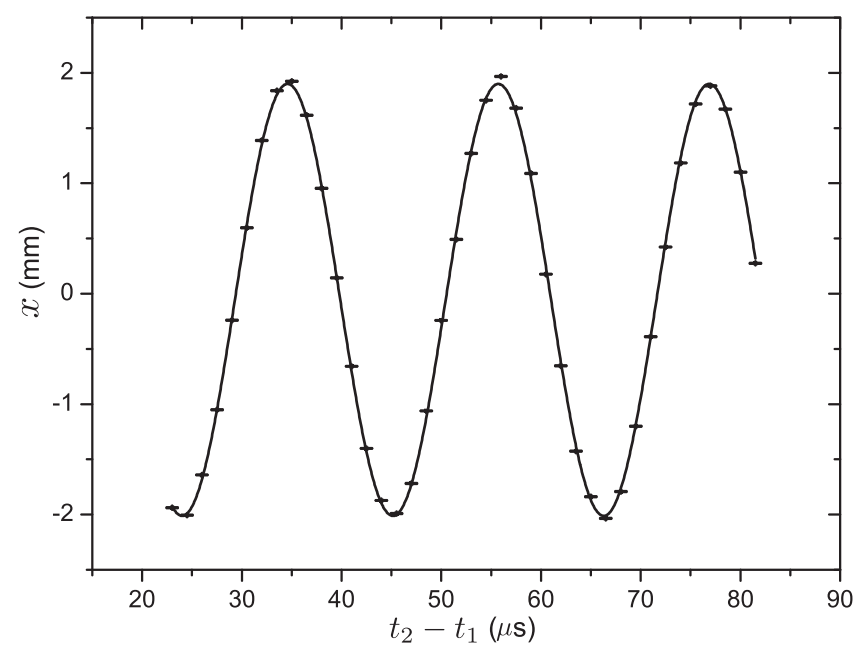

FIG. 3. Measured $x$-position versus time after pulsed excitation (see text). The cloud was initially moved by applying a bias voltage of $\pm 0.14 \mathrm{~V}$ to the appropriate electrodes for $15 \mu \mathrm{s}$. The solid line is a sinusoidal fit to the data. 


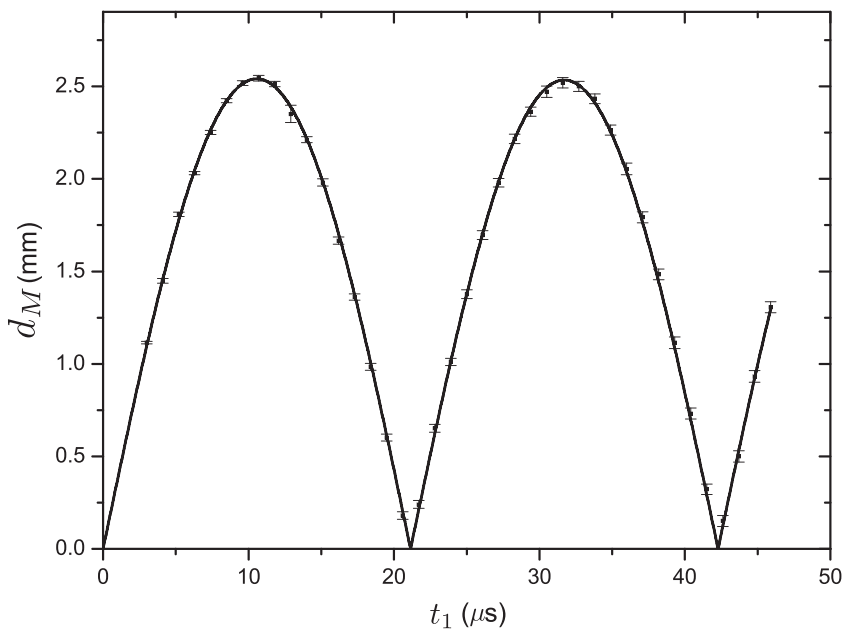

FIG. 4. Displacement, $d_{M}$, as a function of the time following application of the bias voltage $V_{b}= \pm 0.14 \mathrm{~V}$ at $t=0$. The solid line is fitted using Eq. (7) giving $k=1.254 \pm 0.002 \mathrm{~mm}$ and $\omega_{-} / 2 \pi=47.31 \pm 0.03 \mathrm{kHz}$.

observed during RW frequency scans. The measured magnetron frequencies were found to vary with the cloud radial position over a range of $2 \mathrm{kHz}$, which is a consequence of the non-ideal confining potential of our trap.

In Figure 4, the measured displacement, obtained via the method above, is plotted for a range of times $t_{1}$, where the solid line is a fit to Eq. (7). The response of the peak amplitude of the motion to the applied pulse magnitude was found to be linear - as illustrated in Figure 5-with a fitted gradient of $18.5 \pm 0.4 \mathrm{~mm} \mathrm{~V}^{-1}$. From Eq. (7), the peak magnetron radius for a given bias is $2 k=4 a / \omega_{z}^{2}$. If we approximate the electric potential in the trap by parallel plates separated by a distance $D$ and an applied bias of $\pm V_{b}$, then $\phi_{p}=2 V_{b} x / D$. Substituting $\phi_{p}$ for the second term of Eq. (1) gives $a$ $=2 q V_{b} / m D$, hence the expected gradient is simply $2 \mathrm{~d} k / \mathrm{d} V_{b}$ $=8 q / \omega_{z}^{2} m D$. Assuming $D=0.04 \mathrm{~m}$ and $\omega_{z}=60 \mathrm{Mrad} \mathrm{s}^{-1}$ yields $9.6 \mathrm{~mm} \mathrm{~V}^{-1}$; when corrected for adiabatic expansion (caused by the magnetic field differences between the trap and the phosphor screen position), this corresponds to an anticipated $25 \mathrm{mmV}^{-1}$ at the MCP. This is in reasonable

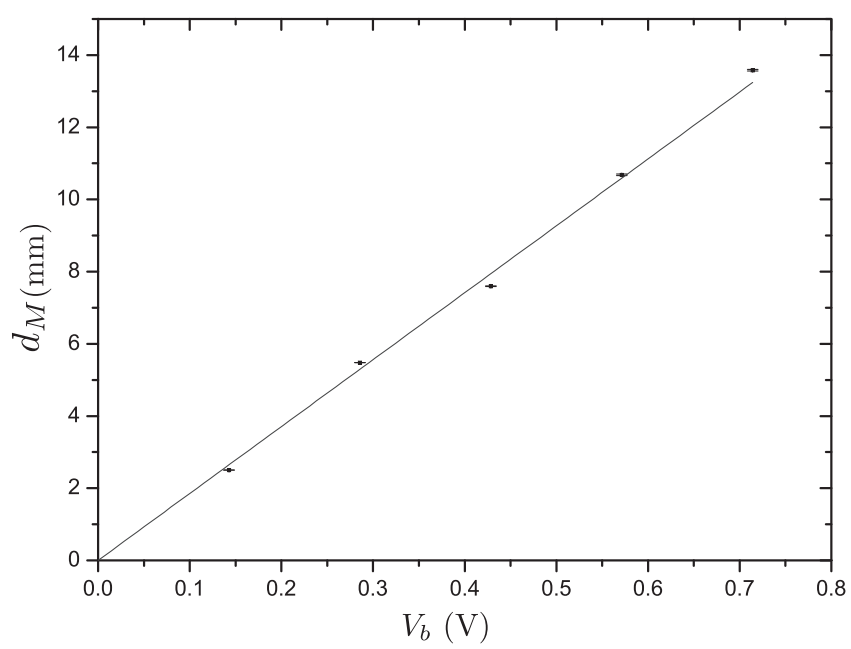

FIG. 5. Peak displacement of the cloud as measured at the MCP as a function of the applied bias voltage. The solid line is a linear fit to the data points with a gradient of $18.5 \pm 0.4 \mathrm{~mm} \mathrm{~V}^{-1}$. accord with the measured value considering the approximations involved, and that the expression for the ideal potential is only valid close to the centre of the trap.

\section{CONCLUSION}

The method described herein enables the magnetron radius of a cloud of ions to be tuned such that it becomes many times larger than the cloud width and in a manner which is independent of the trapped species. The reproducibility of the technique and stability of the motion provide a means to make accurate measurement of the magnetron frequency, which was found to be in reasonable agreement with theoretical estimations and auxiliary measurements. The technique also makes it possible for the position of a cloud to be simply adjusted without alignment of steering magnets or similar deflection devices. This has applications for systems where the particles are required to reach several discrete locations, for instance in multicell trapping, or for soft landing proteins into microarrays. ${ }^{38}$

\section{ACKNOWLEDGMENTS}

We thank the EPSRC for its support of our programme, currently via awards EP/E048951/1 and EP/H026932/1, and the Australian National University for providing JRM with a Vice Chancellor's Travel Grant award. We also like to thank the technical staff of the Physics Department at Swansea University for their enthusiastic support, James Danielson for useful discussions, and Francis Robicheaux for providing us with a self-consistent potential solver.

${ }^{1}$ M. Amoretti, C. Amsler, G. Bonomi, A. Bouchta, P. Bowe, C. Carraro, C. L. Cesar, M. Charlton, M. J. T. Collier, M. Doser, V. Filippini, K. S. Fine, A. Fontana, M. C. Fujiwara, R. Funakoshi, P. Genova, J. S. Hangst, R. S. Hayano, M. H. Holzscheiter, L. V. Jørgensen, V. Lagomarsino, R. Landua, D. Lindelöf, E. L. Rizzini, M. Macrí, N. Madsen, G. Manuzio, M. Marchesotti, P. Montagna, H. Pruys, C. Regenfus, P. Riedler, J. Rochet, A. Rotondi, G. Rouleau, G. Testera, A. Variola, T. L. Watson, and D. P. van der Werf (ATHENA Collaboration), Nature 419, 456 (2002).

${ }^{2}$ G. Gabrielse, N. S. Bowden, P. Oxley, A. Speck, C. H. Storry, J. N. Tan, M. Wessels, D. Grzonka, W. Oelert, G. Schepers, T. Sefzick, J. Walz, H. Pittner, T. W. Hänsch, and E. A. Hessels (ATRAP Collaboration), Phys. Rev. Lett. 89, 233401 (2002).

${ }^{3}$ M. H. Holzscheiter, M. Charlton, and M. M. Nieto, Phys. Rep. 402, 1 (2004).

${ }^{4}$ A. G. Marshall, Int. J. Mass Spectrom. 200, 331 (2000).

${ }^{5}$ L. S. Brown and G. Gabrielse, Rev. Mod. Phys. 58, 233 (1986).

${ }^{6}$ D. H. E. Dubin and T. M. O’Neil, Rev. Mod. Phys. 71, 87 (1999).

${ }^{7}$ X.-P. Huang, F. Anderegg, E. M. Hollmann, C. F. Driscoll, and T. M. ONeil, Phys. Rev. Lett. 78, 875 (1997).

${ }^{8}$ F. Anderegg, E. M. Hollmann, and C. F. Driscoll, Phys. Rev. Lett. 81, 4875 (1998).

${ }^{9}$ R. G. Greaves and C. M. Surko, Phys. Rev. Lett. 85, 1883 (2000).

${ }^{10}$ J. R. Danielson and C. M. Surko, Phys. Rev. Lett. 94, 035001 (2005).

${ }^{11}$ J. R. Danielson, C. M. Surko, and T. M. O'Neil, Phys. Rev. Lett. 99, 135005 (2007).

${ }^{12}$ R. G. Greaves and J. M. Moxom, Phys. Plasmas 15, 072304 (2008).

${ }^{13}$ C. A. Isaac, C. J. Baker, T. Mortensen, D. P. van der Werf, and M. Charlton, Phys. Rev. Lett. 107, 033201 (2011).

${ }^{14}$ D. P. van der Werf, C. A. Isaac, C. J. Baker, T. Mortensen, S. J. Kerrigan, and M. Charlton, New J. Phys. 14, 075022 (2012).

${ }^{15}$ L. V. Jørgensen, M. Amoretti, G. Bonomi, P. D. Bowe, C. Canali, C. Carraro, C. L. Cesar, M. Charlton, M. Doser, A. Fontana, M. C. Fujiwara, R. Funakoshi, P. Genova, J. S. Hangst, R. S. Hayano, A. Kellerbauer, V. Lagomarsino, R. Landua, E. Lodi Rizzini, M. Macrí, N. Madsen, 
D. Mitchard, P. Montagna, A. Rotondi, G. Testera, A. Variola, L. Venturelli, D. P. van der Werf, and Y. Yamazaki (ATHENA Collaboration), Phys. Rev. Lett. 95, 025002 (2005).

${ }^{16}$ R. Funakoshi, M. Amoretti, G. Bonomi, P. D. Bowe, C. Canali, C. Carraro, C. L. Cesar, M. Charlton, M. Doser, A. Fontana, M. C. Fujiwara, P. Genova, J. S. Hangst, R. S. Hayano, L. V. Jørgensen, A. Kellerbauer, V. Lagomarsino, R. Landua, E. Lodi Rizzini, M. Macrí, N. Madsen, G. Manuzio, D. Mitchard, P. Montagna, L. G. C. Posada, A. Rotondi, G. Testera, A. Variola, L. Venturelli, D. P. van der Werf, Y. Yamazaki, and N. Zurlo (ATHENA Collaboration), Phys. Rev. A 76, 012713 (2007).

${ }^{17}$ G. B. Andresen, W. Bertsche, P. D. Bowe, C. C. Bray, E. Butler, C. L. Cesar, S. Chapman, M. Charlton, J. Fajans, M. C. Fujiwara, R. Funakoshi, D. R. Gill, J. S. Hangst, W. N. Hardy, R. S. Hayano, M. E. Hayden, R. Hydomako, M. J. Jenkins, L. V. Jørgensen, L. Kurchaninov, R. Lambo, N. Madsen, P. Nolan, K. Olchanski, A. Olin, A. Povilus, P. Pusa, F. Robicheaux, E. Sarid, S. Seif El Nasr, D. M. Silveira, J. W. Storey, R. I. Thompson, D. P. van der Werf, J. S. Wurtele, and Y. Yamazaki (ALPHA Collaboration), Phys. Rev. Lett. 100, 203401 (2008).

${ }^{18}$ G. B. Andresen, M. D. Ashkezari, M. Baquero-Ruiz, W. Bertsche, P. D. Bowe, E. Butler, C. L. Cesar, S. Chapman, M. Charlton, A. Deller, S. Eriksson, J. Fajans, T. Friesen, M. C. Fujiwara, D. R. Gill, A. Gutierrez, J. S. Hangst, W. N. Hardy, M. E. Hayden, A. J. Humphries, R. Hydomako, M. J. Jenkins, S. Jonsell, L. V. Jørgensen, L. Kurchaninov, N. Madsen, S. Menary, P. Nolan, K. Olchanski, A. Olin, A. Povilus, P. Pusa, F. Robicheaux, E. Sarid, S. Seif El Nasr, D. M. Silveira, C. So, J. W. Storey, R. I. Thompson, D. P. van der Werf, J. S. Wurtele, and Y. Yamazaki (ALPHA Collaboration), Nature 468, 673 (2010).

${ }^{19}$ G. B. Andresen, M. D. Ashkezari, M. Baquero-Ruiz, W. Bertsche, P. D. Bowe, E. Butler, C. L. Cesar, M. Charlton, A. Deller, S. Eriksson, J. Fajans, T. Friesen, M. C. Fujiwara, D. R. Gill, A. Gutierrez, J. S. Hangst, W. N. Hardy, R. S. Hayano, M. E. Hayden, A. J. Humphries, R. Hydomako, S. Jonsell, S. L. Kemp, L. Kurchaninov, N. Madsen, S. Menary, P. Nolan, K. Olchanski, A. Olin, P. Pusa, C. Ø. Rasmussen, F. Robicheaux, E. Sarid, D. M. Silveira, C. So, J. W. Storey, R. I. Thompson, D. P. van der Werf, J. S. Wurtele, and Y. Yamazaki (ALPHA Collaboration), Nat. Phys. 7, 558 (2011).

${ }^{20}$ Y. Enomoto, N. Kuroda, K. Michishio, C. H. Kim, H. Higaki, Y. Nagata, Y. Kanai, H. A. Torii, M. Corradini, M. Leali, E. Lodi-Rizzini, V. Mascagna, L. Venturelli, N. Zurlo, K. Fujii, M. Ohtsuka, K. Tanaka, H. Imao, Y. Nagashima, Y. Matsuda, B. Juhász, A. Mohri, and Y. Yamazaki, Phys. Rev. Lett. 105, 243401 (2010).

${ }^{21}$ C. Amole, M. D. Ashkezari, M. Baquero-Ruiz, W. Bertsche, P. D. Bowe, E. Butler, A. Capra, C. L. Cesar, M. Charlton, A. Deller, P. H. Donnan, S. Eriksson, J. Fajans, T. Friesen, M. C. Fujiwara, D. R. Gill, A. Gutierrez, J. S. Hangst, W. N. Hardy, M. E. Hayden, A. J. Humphries, C. A. Isaac, S.
Jonsell, L. Kurchaninov, A. Little, N. Madsen, J. T. K. McKenna, S. Menary, S. C. Napoli, P. Nolan, K. Olchanski, A. Olin, P. Pusa, C. Ø. Rasmussen, F. Robicheaux, E. Sarid, C. R. Shields, D. M. Silveira, S. Stracka, C. So, R. I. Thompson, D. P. van der Werf, and J. S. Wurtele (ALPHA Collaboration), Nature 483, 439 (2012).

${ }^{22}$ N. Kuroda, H. A. Torii, M. Shibata, Y. Nagata, D. Barna, M. Hori, D. Horváth, A. Mohri, J. Eades, K. Komaki, and Y. Yamazaki, Phys. Rev. Lett. 100, 203402 (2008).

${ }^{23}$ H. Knudsen, H. A. Torii, M. Charlton, Y. Enomoto, I. Georgescu, C. A. Hunniford, C. H. Kim, Y. Kanai, H.-P. E. Kristiansen, N. Kuroda, M. D. Lund, R. W. McCullough, K. Tökesi, U. I. Uggerhøj, and Y. Yamazaki, Phys. Rev. Lett. 105, 213201 (2010).

${ }^{24}$ D. B. Cassidy and A. P. Mills, Jr., Nature 449, 195 (2007).

${ }^{25}$ D. B. Cassidy, V. E. Meligne, and A. P. Mills, Jr., Phys. Rev. Lett. 104, 173401 (2010).

${ }^{26}$ C. M. Surko, J. R. Danielson, and T. R. Weber, "Accumulation, storage and manipulation of large numbers of positrons in traps II-Selected topics," in Physics with Many Positrons, Proc. Int. School of Physics Enrico Fermi, Course CLXXIV, edited by A. Dupasquier, A. P. Mills, Jr., and R. Brusa (IOS Press, Amsterdam, 2010), p. 545.

${ }^{27} \mathrm{~J}$. Xu, M. H. Weber, and K. G. Lynn, "The simulation of moderated positrons behaviour in a micro-trap with long aspect ratio," in Physics with Many Positrons, Proc. Int. School of Physics Enrico Fermi, Course CLXXIV, edited by A. Dupasquier, A. P. Mills, Jr., and R. Brusa (IOS Press, Amsterdam, 2010), p. 575.

${ }^{28}$ T. J. Murphy and C. M. Surko, Phys. Rev. A 46, 5696 (1992).

${ }^{29}$ J. Clarke, D. P. van der Werf, B. Griffiths, D. C. S. Beddows, M. Charlton, H. H. Telle, and P. R. Watkeys, Rev. Sci. Instrum. 77, 063302 (2006).

${ }^{30}$ R. C. Davidson, Physics of Nonneutral Plasmas (Imperial College Press, London, 2002).

${ }^{31}$ K. S. Fine and C. F. Driscoll, Phys. Plasmas 5, 601 (1998).

${ }^{32}$ J. Fajans, E. Gilson, and L. Friedland, Phys. Rev. Lett. 82, 4444 (1999).

${ }^{33}$ S. Kim, M. C. Choi, S. Kim, M. Hur, H. S. Kim, J. S. Yoo, G. T. Blakney, C. L. Hendrickson, and A. G. Marshall, Anal. Chem. 79, 3575 (2007).

${ }^{34}$ S. A. Hofstadler, K. A. Sannes-Lowery, and R. H. Griffey, Rapid Commun. Mass Spectrom. 15, 945 (2001).

${ }^{35}$ J. Notte, J. Fajans, R. Chu, and J. S. Wurtele, Phys. Rev. Lett. 70, 3900 (1993).

${ }^{36}$ D. L. Eggleston, C. F. Driscoll, B. R. Beck, A. W. Hyatt, and J. H. Malmberg, Phys. Fluids B 4, 3432 (1992).

${ }^{37}$ E. Sarid, E. Gilson, and J. Fajans, AIP Conf. Proc. 606, 422 (2002).

${ }^{38}$ Z. Ouyang, Z. Takáts, T. A. Blake, B. Gologan, A. J. Guymon, J. M. Wiseman, J. C. Oliver, V. J. Davisson, and R. G. Cooks, Science 301, 1351 (2003). 